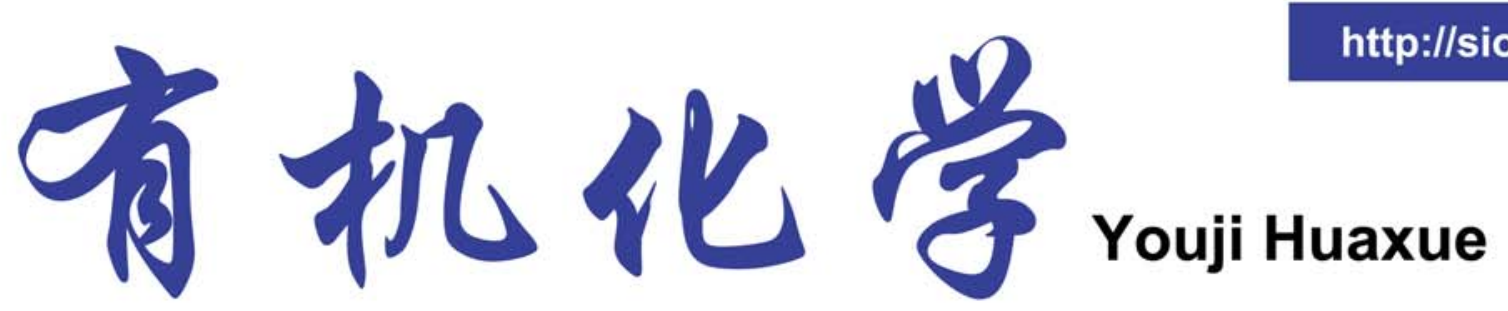 \\ Chinese Journal of Organic Chemistry
}

\section{第33卷 第11期 Vol. 33 No.11 2013}
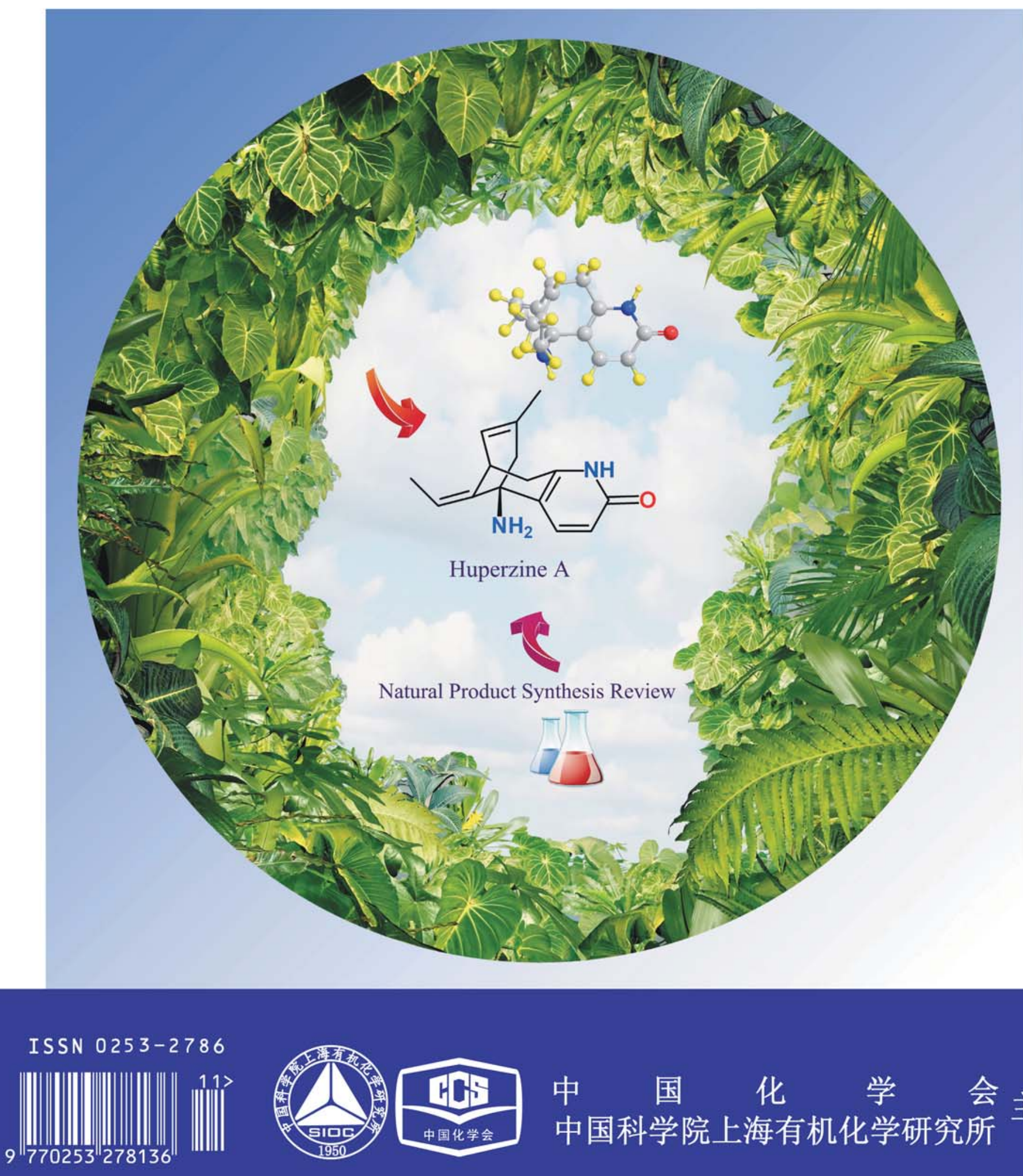

中国 化 学 会 主办
中国科学院上海有机化学研究所 


\section{Born to find out}
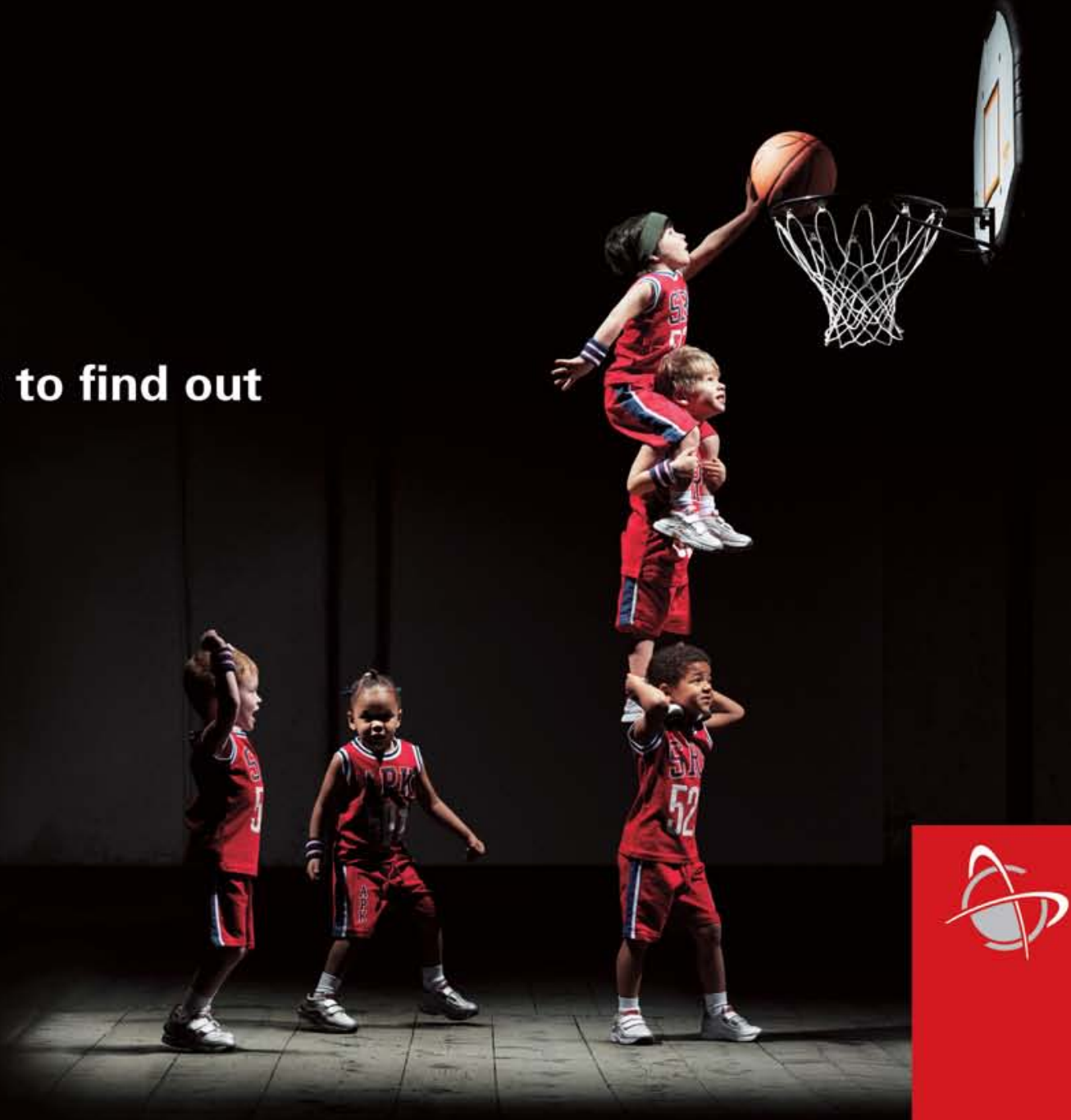

Anton Paar

安东帕 (中国) 有限公司 电话 : 021-6485 5000

传真 : 021-6485 5668

Email: Info.cn@anton-paar.com www.anton-paar.com.cn

\section{安东帕创新分析测试解决方案}

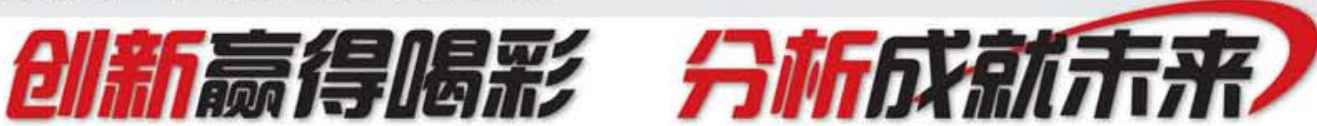

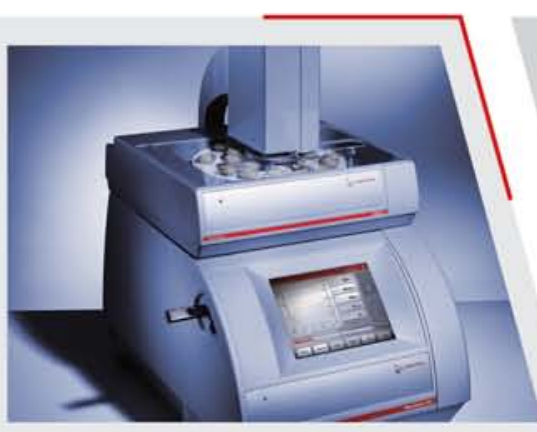

单模微波反应器

Monowave $300+$ MAS 24

一最高微波反应条件: $300^{\circ} \mathrm{C} @ 30 \mathrm{bar}$

一特殊的碳化硅反应管可用于微波机理研究

一实际反应温度和红外温度可同时监控

一叠加式自动进样 - 节省实验室空间

新药研发、金属催化、固相合成及纳米材 料合成等合成反应的方法开发 ( $\mathrm{mg}$ to $\mathrm{g}$ )

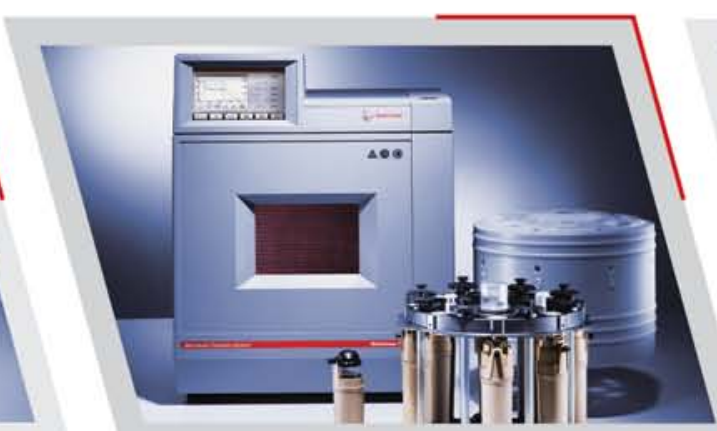

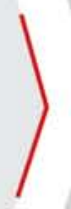

高性能多模微波反应系统

Multiwave PRO

- 可靠的温度和压力传感器 - 最佳反应控制

最高微波反应条件 $\left(300^{\circ} \mathrm{C}\right.$ 和 $\left.80 \mathrm{bar}\right)$ - 拓展了 微波合成的应用范围

一专业的充气装置 一 轻松实现情性气体保护

模块化扩展功能 - 同一主机适用所有类型转子

新药研发、生物医学科学、纳米材料研究、法医鉴 定学等合成的放大反应 ( $\mathrm{g}$ to $\leq 100 \mathrm{~g}$ )

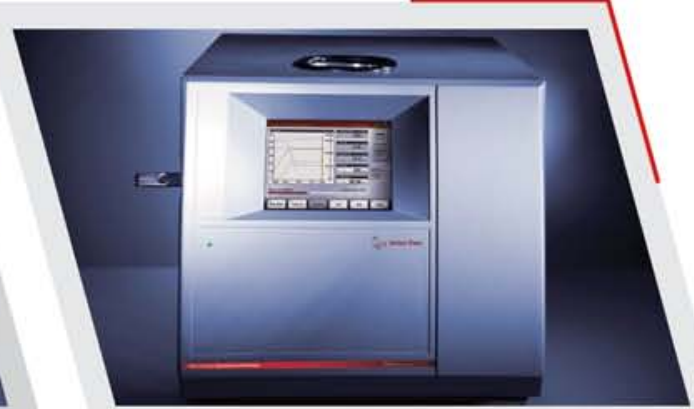

公斤级微波反应器

Masterwave BTR

一最高微波反应条件: $250^{\circ} \mathrm{C} @ 30 \mathrm{bar}$ - 无可比拟的生产力: 公斤级产量

磁力驱动浆式摚拌装置可自动调整搅拌速度 独特冷却安全系统保证公斤级反应安全

新药研发、有机杂环合成、金属催化、固相合成及 纳米材料合成等合成反应的公斤级放大 $(50-300 \mathrm{~g})$ 


\title{
4,5-二取代的菲和 9,10-二氢菲衍生物的合成及液晶性能研究
}

\author{
曹建华*,a,b 李 敏 ${ }^{a, b}$ 隋 岩 ${ }^{a, b}$ 华瑞茂 ${ }^{a, b, c}$ \\ ( ${ }^{a}$ 石家庄诚志永华显示材料有限公司北京研发中心 北京 100084) \\ ( ${ }^{b}$ 河北省平板显示材料工程技术研究中心 石家庄 050091) \\ (c 清华大学化学系 北京 100084)
}

\begin{abstract}
摘要 以 2-氨基-3-取代的苯甲酸为起始原料, 经重氮化-自偶联反应、酯化、还原、氧化、环合反应制备了 4,5-二取代 的菲衍生物，并以还原后的联苯中间体为原料经澳化、格氏化反应制备了 4,5-二取代的 9,10-二氢菲衍生物，以 4,5-二 取代的菲和 4,5-二取代的 9,10-二氢菲衍生物为原料经过卤化、偶联反应制备了液晶单体化合物. 产物结构经过了 ${ }^{1} \mathrm{H}$ $\mathrm{NMR},{ }^{13} \mathrm{C}$ NMR 的确证, 并对其性能进行了测试. 研究结果表明, 这类液晶具有较大的光学各向异性和介电各向异性. 关键词 2-氨基-3-取代的苯甲酸; 菲; 9,10-二氢菲; 液晶; 铁电液晶; 合成
\end{abstract}

\section{Synthesis and Liquid Crystal Property of 4,5-Disubstituted Phenanthrene and 9,10-Dihydrophenanthrene Derivatives}

\author{
Cao, Jianhua ${ }^{*, a, b}$ \\ Li, $\operatorname{Min}^{a, b}$ \\ Sui, Yan ${ }^{a, b}$ \\ Hua, Ruimao ${ }^{a, b, c}$ \\ ( ${ }^{a}$ Beijing Research Center of Shijiazhuang Chengzhi Yonghua Display Material Co. Ltd., Beijing 100084) \\ ( ${ }^{b}$ Hebei Engineering \& Technology Center for Flat Panel Display Material, Shijiazhuang 050091) \\ ( ${ }^{c}$ Department of Chemistry, Tsinghua University, Beijing 100084)
}

\begin{abstract}
Using 2-amino-3-substituted benzoic acid as starting material, 4,5-disubstituted phenanthrene derivatives were synthesized via sequential reactions of diazotization-coupling reaction, esterification, reduction, oxidation and cyclization, and using reduced biphenyl intermediates as starting materials. 4,5-Disubstituted 9,10-dihydrophenanthrene derivatives were synthesized via sequential reactions of bromination, grinard reaction, using 4,5-disubstituted phenanthrene and 4,5-disubstituted 9,10-dihydrophenanthrene derivatives as starting materials. Liquid crystal monomer compounds were synthesized via sequential reactions of halogenations and coupling reaction, and the chemical structures of products were identified by ${ }^{1} \mathrm{H} N M R,{ }^{13} \mathrm{C}$ NMR spectra.

Keywords 2-amino-3-substituted benzoic acid; phenanthrene; 9,10-dihydrophenanthrene; liquid crystal; ferroelectric liquid crystal; synthesis
\end{abstract}

铁电液晶自 1989 年首次提出以来, 在显示领域得 到了广泛的应用, 当前, 新的铁电液晶合成研究报告五 彩纷呈, 但真正有实用价值的并不多, 作为液晶化合物, 除了要求对热、电化学、光学性能稳定性以及在室温附 近有较宽的相变温度外, 还要求具有小的光学各向异性 $\Delta n$ 值和小的最好是正的或者大的负介电各向异性 $\Delta \varepsilon$ 值. 除了向列型和胆甾液晶, 现在已经出现了光学活性的倾 斜近晶液晶用于商业的显示设备中, 然而由于铁电性液 晶具有分子层状排列结构, 在相变过程中分子层容易发
生倾斜，破坏器件的光学效果，使铁电液晶器件无法产 业化. 但铁电液晶分子的偶极矩比向列相液晶分子大得 多, 因此电光响应速度比向列相液晶快 $1 \sim 2$ 个数量级, 响应时间约数十微秒到百微秒级, 一直是广大科研工作 者关注的重点. 在光电组件中, 要获得一个良好的对比 度, 需要一个统一的液晶平面方向, 在 $S_{\mathrm{A}}$ 和 $S_{\mathrm{C}}$ 相要实 现好的液晶取向, 就要求液晶材料必须具有低的粘度和 较高的自发极化以获得短的响应时间. 基于显示器件的 使用要求, 液晶化合物应具有适宜的光学各向异性、介

\footnotetext{
*E-mail: talentona@163.com

Received June 20, 2013; revised July 15, 2013; published online July 26, 2013.

Project supported by the National Natural Science Foundation of China (No. 21032004), the Specialized Research Fund for the Doctoral Program of Higher Education of China (No. 20110002110051) and the National Electronics and Information Industry Development Fund.

国家自然科学基金(No. 21032004)、高等学校博士学科点专项科研基金(No. 20110002110051)和国家电子和信息产业发展基金资助项目.
} 
电各向异性、粘度、弹性常数比值、电阻率、宽的液晶 相态温度范围、以及良好的互溶性. 侧向氟取代的液晶 化合物具有大负数的介电各向异性 $\Delta \varepsilon$ 值, 但由于氟取 代的相邻两个苯环在氟取代基的影响下能自由旋转 ${ }^{[1]}$, 氟取代基不能固定保持在同侧, 不能自发取向, 需要在 外加电场的作用下旋转至同侧, 这在一定程度上降低了 液晶分子的响应时间. 基于以上思路我们设计和合成了 4,5-二氟取代的和 4,5-双(三氟甲基)取代的菲和 9,10-二 氢菲衍生物, 使氟取代或三氟甲基取代的相邻两个苯环 给以固定. 图 1 所示为邻位二取代联苯结构示意图.
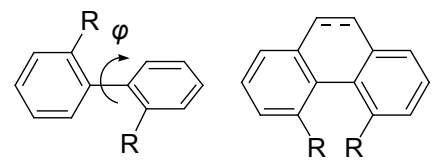

图 1 邻位二取代联苯结构示意图

Figure 1 Structure diagram of ortho disubstituted biphenyl

菲、9,10-二氢菲以及 $1,2,3,4$-四氢菲衍生物作为液 晶化合物已经在很多的专利中有描述, 由于其具有负的 介电各向异性 $\Delta \varepsilon$ 值, 而作为铁电液晶使用, 但是均没有 相关描述固定住 4, 5 位两个位置的取代基化合物, 也没 有相关的液晶性质研究的文献报道, 而作为菲或 9,10二氢菲的合成方法却有很多相关报道 ${ }^{[2 \sim 9]}$, 我们在文献 的基础上设计了关键化合物的合成路线(图 2 和图 3), 并 从关键中间体出发, 合成了数个液晶单体, 并考查了它 们的变相温度以及在偏光显微镜下观查它们的液晶温 度范围.

\section{1 结果与讨论}

\section{1 合成讨论}

4,5-二取代的-9,10-二氢菲的合成方法 ${ }^{[10]}$ 是以 2-氨 基-3- $\mathrm{R}^{1}\left(\mathrm{~F}, \mathrm{CF}_{3}\right)$-苯甲酸 $(6)$ 为原料, 经重氮盐在铜离子催 化下偶合得到 6,6'-二取代的联苯-2,2'-二甲酸甲酯(13),
联苯化合物 13 在低温下用氢化铝锂或在四氢呋喃一甲醇 回流状态下用嗍氢化钠还原为二苠醇 14, 茮醇 14 用三 浿氧膦溴化得到联苯二溴茮 $\mathbf{1 5}$, 而溴茮的格式化偶联 反应只需在较低的温度下即可顺利进行, 得到关键的中 间体 4,5-二取代的-9,10-二氢菲(16)(图 2, 合成路线 1). 在无水三氯化铁或磷酸三甲酯催化下溴化 16 可选择性 得到单溴代的 $17^{[11]}$ 和双溴代的 $18^{[12]}$, 单溴代的 17 和原 料 16 无法分离纯化，而 18 和 17 可通过重结晶分离.

4,5-二取代的菲的合成法 ${ }^{[14,15]}$ 是以 2 -氨基-3- $\mathrm{R}^{1}(\mathrm{~F}$, $\mathrm{CF}_{3}$ )-苯甲酸 $(6)$ 为原料，在氯仿溶液中溴化制备 $5^{[16]}$. 它 以氢溴酸盐的形式沉淀出来, 不需要纯化即可重氮化. 在铜盐催化下顺利制备了联苯 $4 \mathbf{a}$, 但由于氨基对位溴 的影响, 由 5 重复数次未能合成出 $\mathbf{4 b}$, 在用氢化铝锂还 原 $4 \mathrm{a}$ 的过程中, 意外发现可定量消除一个溴制备化合 物 10(图 3, 合成路线 2). 研究了氢化铝锂的投料配比以 及反应温度对产物 10 收率的影响. 将氢化铝锂换成硼 氢化钠后, 在四氢呋喃一甲醇溶液中回流反应可高收率 的得到二苠醇 $3 \mathrm{a}$ ，二芐醇化合物 10 和 $3 \mathrm{a}$ 用温和的 Swern 氧化剂 ${ }^{[17]}$ 即可氧化为联苯二醛 11a 和 $2 \mathbf{a}, 11 \mathbf{a}$ 和 $2 \mathbf{a}$ 在乙酸回流条件下滴加过量的水合肼，即可关环得 到单溴代的菲 12a 或双溴代的 $1 \mathbf{a}^{[18,19]}$

4,5-双 (三氟甲基)菲(1b， 12b) 的合成可由相应的 18b，17b 用 DDQ (2,3-二氯-5,6-二氰基对苯醌)氧化脱 氢来制备 ${ }^{[20]}$. 通过上述合成路线 1 和路线 2 分别制备出 了关键的中间体 $17,18,12 \mathrm{a}, 1 \mathrm{a}$ 以及 $1 \mathrm{~b}$ 和 $12 \mathrm{~b}$, 再与不 同的基团反应合成出不同系列的液晶单体分子，见图 4 .

\section{2 氢化铝锂的用量和反应温度对还原反应的影响}

以 4,4'-二溴-6,6'-二氟联苯-2,2'-二甲酸甲酯 $(4 \mathbf{a})^{[21]}$ 为反应底物可选用四氢呋喃为溶剂, 分批加入氢化铝 锂，在不同的反应温度下还原，考查了温度对消除反应 的影响, 实验结果见表 1 .
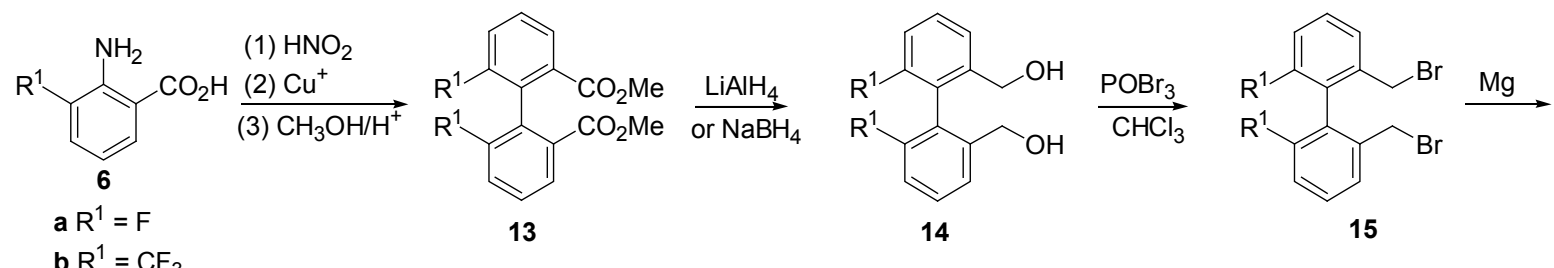<smiles>[R]c1cccc2c1-c1c([R])cccc1CC2</smiles>

16
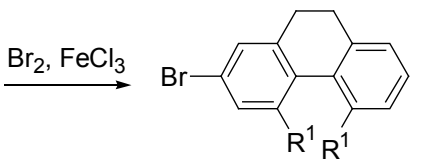

17

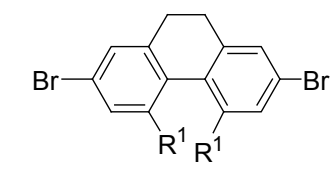

18

图 2 合成路线 1

Figure 2 Synthesis of route 1 


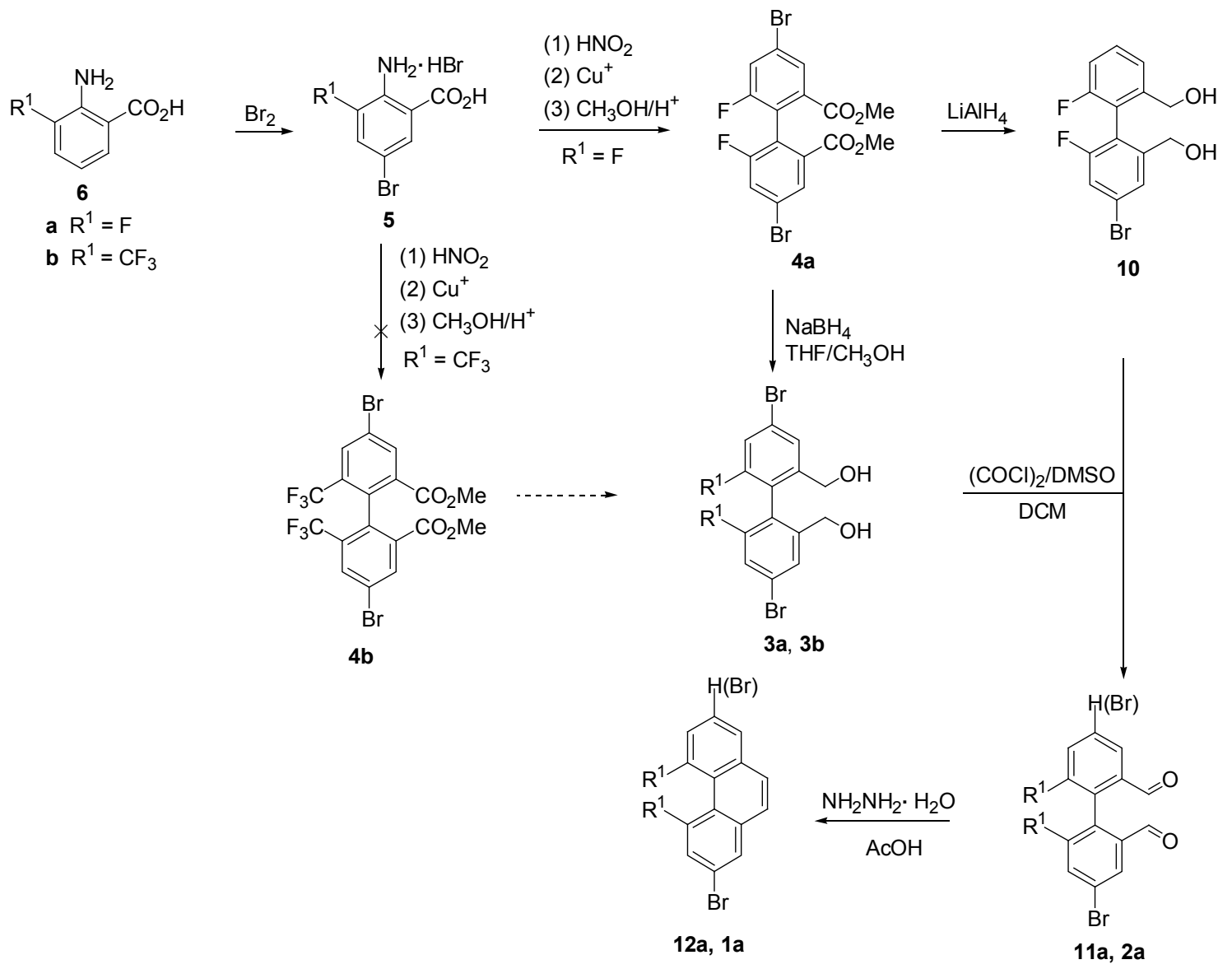

图 3 合成路线 2

Figure 3 Synthesis of route 2<smiles></smiles>

$1,12,17,18$ a $R^{1}=F$ b $\mathrm{R}^{1}=\mathrm{CF}_{3}$ c Z $=\mathrm{CH}_{2} \mathrm{CH}_{2}$ d $\mathrm{Z}=\mathrm{CH}=\mathrm{CH}$ $\mathrm{R}^{2}=\mathrm{H}, \mathrm{Br}$ $\mathrm{R}^{3}=\mathrm{C}_{n} \mathrm{H}_{2 n+1}$<smiles>[R]c1cc([R])c2c([Z]c3cc([Y19])cc([R])c32)c1</smiles>

19<smiles>[Y]c1cc([R])c2c([Y]c3cc([Y])cc([R])c32)c1</smiles>

20<smiles>[R]C1CCC(=O)CC1</smiles>

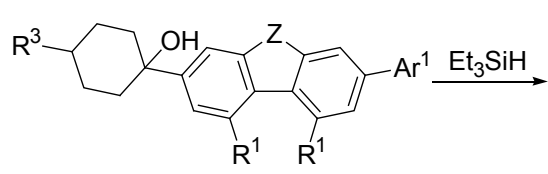

21<smiles>[R]C=Cc1cc([R])c2c([Y]c3cc([Al])cc([R])c32)c1</smiles>

22

19ad 19_1 $\mathrm{Ar}^{1}=3,4,5$-trifluorophenyl, $\mathrm{Ar}^{2}=\mathrm{H}$; 19_2 $\mathrm{Ar}^{1}=$ 2,3-difluoro-4-propylphenyl, $\mathrm{Ar}^{2}=\mathrm{H}$

20ad 20_1 $\mathrm{Ar}^{1}=3,4,5$-trifluorophenyl, $\mathrm{Ar}^{2}=4$-(4-propylcyclohexyl)pheny; 20_2 $\mathrm{Ar}^{1}=3,5$-difluoro-4-(trifluoromethoxy)phenyl, $\mathrm{Ar}^{2}=$ 2,3-difluoro-4-propylphenyl; 20_3 $\mathrm{Ar}^{1}=3,4,5$-trifluorophenyl, $\mathrm{Ar}^{2}=$ 2-fluoro-4-pentylpheny; 20_4 $\mathrm{Ar}^{1}=$ 2,3-difluoro-4-propylphenyl, $\mathrm{Ar}^{2}=2,3$-difluoro-4-propylphenyl

19bc 19_3 $\mathrm{Ar}^{1}=3,4,5$-trifluorophenyl, $\mathrm{Ar}^{2}=\mathrm{H} ; 19 \_4 \mathrm{Ar}^{1}=$ 2,3-difluoro-4-pentylphenyl, $\mathrm{Ar}^{2}=\mathrm{H}$

20bc 20_5 $\mathrm{Ar}^{1}=3,4,5$-trifluorophenyl, $A \mathrm{r}^{2}=$ 2-fluoro-4-pentylphenyl

22ad 22_1 $\mathrm{Ar}^{1}=3,4,5$-trifluorophenyl, $\mathrm{R}^{3}=$ propyl

22bc 22_2 $\mathrm{Ar}^{1}=3,4,5$-trifluorophenyl, $\mathrm{R}^{3}=$ propyl

图 4 合成路线 3

Figure 4 Synthesis of route 3 
表 1 反应温度对还原反应的影响

Table 1 Effect of temperature on the reduction reaction

\begin{tabular}{ccccc}
\hline Entry & LAH/equiv. & Temperature $/{ }^{\circ} \mathrm{C}$ & Time $/ \mathrm{h}$ & Yield $^{a_{0}} \%$ \\
\hline 1 & 2.0 & 0 & 0.5 & 65.0 \\
2 & 2.0 & -5 & 0.5 & 70.5 \\
3 & 2.0 & -10 & 1 & 83.2 \\
4 & 2.0 & -20 & 1 & 94.7 \\
5 & 2.0 & -40 & 3 & 93.5 \\
\hline
\end{tabular}

$a$ 氢化铝锂投入 2 equiv., 以原料 $\mathbf{4 a}$ 为标准计算收率.

由表 1 中可以得出, 反应温度为 $0{ }^{\circ} \mathrm{C}$ 时只得到了 $65 \%$ 的收率, 随着温度的降低, 反应收率逐渐增加, 控 制在 $-20{ }^{\circ} \mathrm{C}$ 时, 产物(4-溴-6,6'-二氟联苯-2,2'-二基)二 甲醇 $(\mathbf{1 0})$ 的收率达到最高, 再降低反应温度也无明显变 化, 反而反应时间变长了, 所以反应温度在 $-20{ }^{\circ} \mathrm{C}$ 最 合适.

由表 2 中可以得出, 在确定了反应温度后, 随着氢 化铝锂的用量由 1.8 equiv. 增加到 2.2 equiv., 反应收率 明显提高, 但超过 2.4 equiv. 后, 收率下降明显, 主要是 消除两个溴的副产物增多了, 所以氢化铝锂的投料配比 控制在 2.4 equiv. 最为合适.

表 2 氢化铝锂的用量对还原反应的影响 ${ }^{a}$

Table 2 Effect of the molar ratio of lithium aluminium hydride on the reduction reaction

\begin{tabular}{ccccc}
\hline Entry & LAH/equiv. & Temperature $/{ }^{\circ} \mathrm{C}$ & Time $/ \mathrm{h}$ & Yield $^{a_{0}} \%$ \\
\hline 1 & 1.8 & -20 & 1 & 72.4 \\
2 & 2.0 & -20 & 1 & 78.7 \\
3 & 2.2 & -20 & 1 & 85.3 \\
4 & 2.4 & -20 & 1 & 94.0 \\
5 & 2.6 & -20 & 1 & 87.8 \\
\hline
\end{tabular}

${ }^{a}$ 表 1 和表 2 内 $\mathrm{LAH}$ 表示氢化铝锂. ${ }^{b}$ 反应温度控制在 $-20{ }^{\circ} \mathrm{C}$, 反应时间 为 $1 \mathrm{~h}$, 以原料 $\mathbf{4 a}$ 为标准计算收率.

\section{2 液晶性质研究}

通过图 4 合成路线 3 的合成，以 $17 \mathbf{a}, 17 \mathbf{b}, 18 \mathbf{a}, 18 b$, $12 \mathrm{a}, 1 \mathrm{a}$ 以及 $1 \mathrm{~b}$ 和 $12 \mathrm{~b}$ 为原料, 合成了 $19 \mathrm{ad}, 20 \mathrm{ad}, 19 \mathrm{bc}$, $20 \mathrm{bc}, 23 \mathrm{ad}, 23 \mathrm{bc}$ 系列单体液晶化合物, 首先用 DSC $822 \mathrm{e}$ 型差热分析扫描量热仪, 加热速率为 $5{ }^{\circ} \mathrm{C} / \mathrm{min}$ 检测了各化合物的相变温度变化. 从表 3 中可以看出, 19_1, 19_2, 19_4, 20_1, 20_2, 20_3,23_1, 23_2 化合物可 能有液晶性能, 图 5 为 20_2 的 DSC 相变图, 通过 $\operatorname{POM}$ (偏光显微镜)观察了各化合物的织构(图 6 为化合 物 $20 \_2$ 的织构), 并与液晶的标准相图对照, 确定了化 合物的相态. 单体液晶化合物的光学各向异性 $(\Delta n)$ 使用 NAR-47 型阿贝折射仪, 采用钠光源 $(589 \mathrm{~nm})$, 在 $20{ }^{\circ} \mathrm{C}$ 下测定. 表 3 为测试得到的几种液晶化合物的相变温度 和光学各向异性(选取石家庄诚志永华液晶显示材料公 司生产的编号为 SLC090105 的商品液晶化合物作为母 体, 将单体液晶化合物以 $5 \%$ 的质量比例溶于母体), 测 得混合物的 $n_{\mathrm{o} 1}$ 和 $n_{\mathrm{e} 1}$, 计算得到光学各向异性 $\Delta n=$ $n_{\mathrm{e} 1}-n_{\mathrm{o} 1}$, 外推算出纯的单体液晶化合物的光学各向异 性 $\Delta n$.

表 3 液晶化合物的相变温度、光学各向异性

Table 3 Optical anisotropy and phase transition temperature of the liquid crystals

\begin{tabular}{|c|c|c|}
\hline 化合物 ${ }^{a}$ & 光学各向异性 $(\Delta n)$ & 相变温度 ${ }^{b} /{ }^{\circ} \mathrm{C}$ \\
\hline 19_1 & 0.411 & $172.68 \sim 205.45$ \\
\hline $19 \_2$ & 0.382 & $149.84 \sim 202.56$ \\
\hline $19 \_3$ & 0.326 & $169.52 \sim 261.62$ \\
\hline $19 \_4$ & 0.259 & $148.37 \sim 262.54$ \\
\hline $20 \_1$ & 0.226 & $123.33 \sim 283.90$ \\
\hline $20 \_2$ & 0.405 & $112.88 \sim 284.40$ \\
\hline $20 \_3$ & 0.305 & $140.51 \sim 225.27$ \\
\hline $20 \_4$ & 0.242 & 80.96 \\
\hline $20 \_5$ & 0.308 & $182.52 \sim 281.61$ \\
\hline 23_1 & 0.314 & $128.63 \sim 152.55$ \\
\hline $23 \_2$ & 0.216 & $147.78 \sim 162.42$ \\
\hline
\end{tabular}

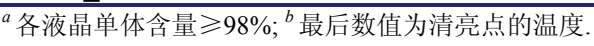

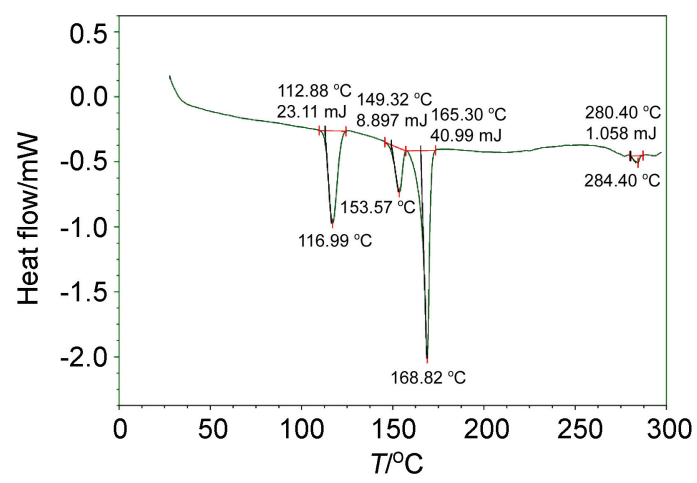

图 5 化合物 20_2 的相变图(DSC)

Figure 5 Phase transition of compound 20_2

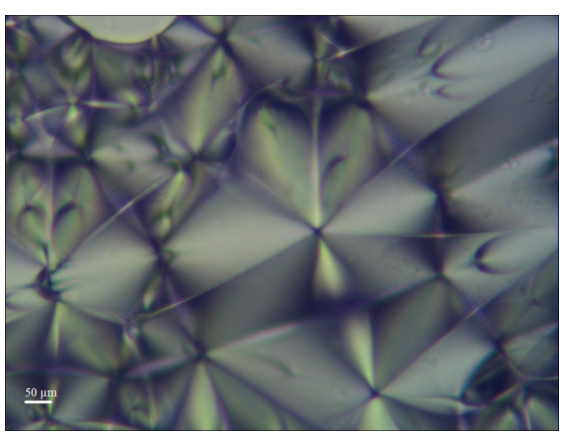

图 6 化合物 20_2 的织构

Figure 6 Texture of compound 20_2

\section{2 结论}

以 2-氨基-3-氟苯甲酸和 2-氨基-3-三氟甲基苯甲酸 为起始原料，经过溴化、重氮化、酯化、还原、氧化、 格氏化等反应合成了 4,5-二氟菲、4,5-二(三氟甲基)菲、 4,5-二氟-9,10-二氢菲、4,5-二(三氟甲基)- 9,10 -二氢菲的 衍生物 19ad, 20ad, 19bc, 20bc, 23ad, 23bc, 并通过 ${ }^{1} \mathrm{H}$ $\mathrm{NMR}$ 和 ${ }^{13} \mathrm{C} \mathrm{NMR}$ 确证合成出的化合物的正确性，部分 
化合物在偏光显微镜下观察其织构, 显示出了液晶相.

\section{3 实验部分}

\section{1 仪器与试剂}

实验中所用仪器设备主要有 DSC822e 型差热分析 扫描仪; ${ }^{1} \mathrm{H}$ NMR 和 ${ }^{13} \mathrm{C}$ NMR 由 Bruker 300 型核磁共振 仪测定 (TMS 为内标, $\mathrm{CDCl}_{3}$ 为溶剂); GC-MS 由 $\mathrm{Hp}$ 5890-5972 型气质谱联用仪测定; 光学各向异性由 NAR-47 型阿贝折射仪测定. 所用芳基硼酸均为石家庄 诚志永华液晶显示材料公司自己生产, 其他试剂均为市 售分析纯.

\section{2 实验方法}

3.2.12-氨基-3-氟-5-溴苯甲酸氢溴酸盐 (5a) 和 2-氨 基-3-(三氟甲基)-5-溴苯甲酸氢溴酸盐 $(5 b)$ 的合成

$153 \mathrm{~g}$ (987 mmol)的 2-氨基-3-氟苯甲酸(6a) 分散在 $1500 \mathrm{~mL}$ 氯仿中, $175 \mathrm{~g}(1.09 \mathrm{~mol})$ 的溴素溶于 $150 \mathrm{~mL}$ 氯 仿的溶液缓慢的滴加到上述氯仿溶液中, 并于室温搅拌 反应过夜, 次日过滤, 滤饼用二氯甲烷洗涤, 真空干燥 后得 $276 \mathrm{~g}$ 的 2-氨基-3-氟-5-溴苯甲酸氢溴酸盐 $5 \mathrm{a}$, 黄色 粉末, 产率 $88.7 \%$, 无需纯化.

2-氨基-3-(三氟甲基)-5-溴苯甲酸氢溴酸盐(5b)的合 成同上操作，黄色粉末，产率 $86 \%$, 无需纯化.

$3.2 .26,6^{\prime}-$ 双 (三氟甲基)联苯- $2,2^{\prime}$-二闸酸甲酯(13b) 和 $4,4^{\prime}$-二溴-6, 6'-二氟联苯-2,2'-二甲酸甲酯 (4a) 的合 成

$75 \mathrm{~g}(0.3 \mathrm{~mol})$ 的五水硫酸铜溶于 $300 \mathrm{~mL}$ 水中, 搅 拌下加入 $230 \mathrm{~mL}$ 的 $25 \%$ 氨水, 用冰盐浴降温至 $0{ }^{\circ} \mathrm{C}$, 将 $20.8 \mathrm{~g}(0.3 \mathrm{~mol})$ 的着胺盐酸盐和 $12.8 \mathrm{~g}(0.32 \mathrm{~mol})$ 的氢 氧化钠配成的水溶液缓慢滴加入上述冷的铜溶液中, 并 保持在 $0{ }^{\circ} \mathrm{C}$ 以下备用.

$41 \mathrm{~g}(0.2 \mathrm{~mol})$ 的 2-氨基-5-溴-3-氟苯甲酸氢溴酸盐 和 $85 \mathrm{~mL}$ 的浓盐酸混合, 加入 $300 \mathrm{~mL}$ 水和 $60 \mathrm{~mL}$ 的乙 腈, 并用冰盐浴降温至 $0{ }^{\circ} \mathrm{C}$ 以下, $15.9 \mathrm{~g}(0.23 \mathrm{~mol})$ 的亚 硝酸钠溶于 $120 \mathrm{~mL}$ 水的溶液缓慢滴加入上述溶液中, 于 $5{ }^{\circ} \mathrm{C}$ 以下搅拌反应 $1 \mathrm{~h}$, 将此清澈的重氮溶液缓慢地 滴加入备用的铜溶液内, 此过程保持在 $10{ }^{\circ} \mathrm{C}$ 以下, 于 室温摚拌反应 $1 \mathrm{~h}$, 加热升温至 $85{ }^{\circ} \mathrm{C}$ 并滴加入浓盐酸酸 化, 冰水浴降温到 $0{ }^{\circ} \mathrm{C}$ 过滤出不溶的固体, 滤饼用水 洗, 真空干燥, 得到 $33.9 \mathrm{~g}$ 的 6,6'-双(三氟甲基)联苯2,2'-二甲酸, 类白色的固体.

将中间体和 $330 \mathrm{~mL}$ 的无水甲醇混合, 加热升温至 回流, 缓慢滴加入 $10 \mathrm{~mL}$ 的氯化亚砜, 回流反应过夜, 得到清亮的溶液减压浓缩干, 残余物用硅胶柱分离, 乙 酸乙酯/石油醚 $(V: V=1: 20 \sim 50)$ 洗脱, 得到 $34.5 \mathrm{~g}$ 的 6,6'-双(三氟甲基)联苯-2,2'-二甲酸甲酯(13b), 白色结晶,
两步总产率 $42 \%$

4,4'-二溴-6,6'-二氟联苯-2,2'-二甲酸甲酯 $(4 a)$ : 白色 结晶, 两步总产率 54\%

$3.2 .3\left[6,6^{\prime}\right.$-双(三氟甲基)联苯- $2,2^{\prime}$-二基] 二甲醇(14b) 的合成

$20 \mathrm{~g}(0.05 \mathrm{~mol}$ )的 6,6'-双 (三氟甲基)联苯-2,2'-二甲 酸甲酯(13b)用 $400 \mathrm{~mL}$ 干燥的 THF 溶解, 将此清亮的溶 液用冰盐浴冷却至 $-5{ }^{\circ} \mathrm{C}$, 缓慢地分批加入 $5.6 \mathrm{~g}(0.14$ $\mathrm{mol}$ )的氢化铝锂, 保温搅拌反应 $1 \mathrm{~h}$, 缓慢升到室温, 搅 拌反应 $12 \mathrm{~h}$, 向反应液内滴加入水和 $17 \mathrm{~mL}$ 的 $15 \%$ 氢氧 化钠水溶液淬灭反应，抽滤，滤饼用 THF 洗，滤液减压 浓缩干, 得到 $15.5 \mathrm{~g}$ 的 [6,6'-双(三氟甲基)联苯-2,2'-二基] 二甲醇(14b), 白色结晶, 产率 $90 \%$, 无需纯化, 继续下 步反应.

3.2 .4 (4-溴-6, $6^{\prime}$-二氟联苯- $2,2^{\prime}$-二基) 二甲醇 (10)的合 成

$35 \mathrm{~g}$ (75.4 mmol)的 4,4'-二溴-6,6'-二氟联苯-2,2'-二 甲酸甲酯(4a)用 $300 \mathrm{~mL}$ 干燥的 THF 溶解, 将此清亮的 溶液用液氮 - 乙醇浴冷却至 $-20{ }^{\circ} \mathrm{C}$, 缓慢地分批加入 7 $\mathrm{g}(184.2 \mathrm{mmol})$ 的氢化铝锂, 保温搅拌反应 $1 \mathrm{~h}$, 缓慢升 到室温, 搅拌反应 $5 \mathrm{~h}$, 向反应液内滴加入水和 $20 \mathrm{~mL}$ 的 $15 \%$ 氢氧化钠水溶液淬灭反应，抽滤，滤饼用 $\mathrm{THF}$ 洗， 滤液减压浓缩干, 得到 $23.5 \mathrm{~g}$ 的(4-溴-6,6'-二氟联苯2,2'-二基)二甲醇(10)，黄色油状物，产率 94.7\%，无需纯 化, 继续下步反应.

3.2 .5 (4, 4'-二溴-6, 6'-二氟联苯-2,2'-二基)二甲醇 $(3 \mathbf{a})$ 的合成

$35 \mathrm{~g}$ (75.4 mmol)的 4,4'-二溴-6,6'-二氟联苯-2,2'-二 甲酸甲酯(4a)用 $300 \mathrm{~mL}$ 的 $\mathrm{THF}$ 以及 $30 \mathrm{~mL}$ 的甲醇搅拌 溶解, 缓慢地分批加入 $10 \mathrm{~g}(263 \mathrm{mmol})$ 的硼氢化钠固体 粉末，加热升温回流搅拌反应 $2 \mathrm{~h}$, 减压浓缩干，向残余 物内加入 $200 \mathrm{~mL}$ 的水搅拌分散, 用乙酸乙酯萃取 3 次, 收集有机相, 并用无水硫酸钠干燥, 抽滤, 滤液减压浓 缩干, 得到 $28 \mathrm{~g}$ 的(4,4'-二溴-6,6'-二氟联苯-2,2'-二基)二

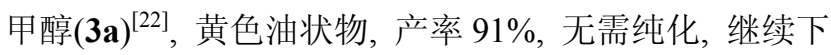
步反应.

$3.2 .62,2^{\prime}-$ 双(溴甲基)-6,6'-双(三氟甲基)联苯(15b)的 合成

$15 \mathrm{~g}$ (42.8 mmol)的(6,6'-双(三氟甲基)联苯-2,2'-二 基)二甲醇(14b)溶于 $250 \mathrm{~mL}$ 的氯仿中, 缓慢分批加入 $49 \mathrm{~g}(0.17 \mathrm{~mol})$ 的三溴氧磷, 升温回流反应 $10 \mathrm{~h}$, 冷却到 室温, 用饱和的食盐水洗 3 次, 收集的有机相用无水硫 酸钠干燥, 过滤, 滤液减压浓缩干, 残余物用短的硅胶 柱脱色, 用石油醚洗脱, 得 $18.3 \mathrm{~g}$ 的 2,2'-双(溴甲 基)-6,6'-双(三氟甲基)联苯(15b)，黄色的油状物，产率 
$89.7 \%$.

\subsubsection{4,5-双(三氟甲基)-9,10-二氢菲(16b)的合成}

$7.8 \mathrm{~g}$ (16.3 mmol)的 2,2'-双(溴甲基)-6,6'-双(三氟甲 基)联苯 $(\mathbf{1 5 b})$ 溶于 $150 \mathrm{~mL}$ 的无水乙醚中, 在氮气保护 下, 加入 $0.7 \mathrm{~g}(29 \mathrm{mmol})$ 镁粉和一粒碘, 加热回流反应 1 $\mathrm{h}$, 在超声下回流反应直至镁粉消失, 冷却到室温, 加入 少量水, 分出乙醚相, 并用饱和食盐水洗 2 次, 有机相 用无水 $\mathrm{MgSO}_{4}$ 干燥, 过滤, 滤液减压浓缩干, 残余物用 硅胶柱分离纯化, 乙酸乙酯/石油醚 $(V: V=1: 20 \sim 50)$ 洗脱, 得 $1.5 \mathrm{~g}$ 的 4,5-双(三氟甲基)-9,10-二氢菲(16b), 白 色固体，产率 $28.9 \%$.

3.2 .8 4-溴-6, $6^{\prime}$-二氟联苯-2,2'-二甲醛(11a)和 $4,4^{\prime}-$ 二 溴-6, ' 1 -二氟联苯- $2,2^{\prime}-$ 二甲醛 $(\mathbf{2 a})$ 的合成

$26.1 \mathrm{~mL}$ 的二甲基亚砜和 $150 \mathrm{~mL}$ 的二氯甲烷混合 均匀, 用液氮浴冷却至 $-78{ }^{\circ} \mathrm{C}$, 缓慢滴加入 $15.6 \mathrm{~mL}$ 的 草酰氯溶于 $100 \mathrm{~mL}$ 的二氯甲烷溶液, 再滴加入 $30 \mathrm{~g}$ (73.5 mmol)的(4,4'-二溴-6,6'-二氟联苯-2,2'-二基)二甲醇 (3a) 溶于二氯甲烷的溶液, 保温搅拌反应 $1 \mathrm{~h}$, 再滴加入 $101.7 \mathrm{~mL}$ 的三乙胺, 搅拌反应 $30 \mathrm{~min}$, 缓慢升到室温, 加入 $150 \mathrm{~mL}$ 的饱和食盐水洗 3 次, 分出有机相, 用无水 $\mathrm{Na}_{2} \mathrm{SO}_{4}$ 干燥, 抽滤, 滤液减压浓缩干, 残余物用 $50 \mathrm{~mL}$ 的无水乙醇重结晶, 得到 $26 \mathrm{~g}$ 的 4,4'-二溴-6,6'-二氟联 苯-2,2'-二甲醛 $(2 \mathrm{a})$, 黄色固体，产率 $87.5 \%$.

4-溴-6,6'-二氟联苯-2,2'-二甲醛(11a), 黄色固体, 产 率 $84 \%$.

3.2 .9 2-溴-4,5-二氟菲(12a)和 2,7-二溴-4,5-二氟菲 (1a)的合成

$18.5 \mathrm{~g}$ (45.8 mmol)的 4,4'-二溴-6,6'-二氟联苯-2,2'二甲醛(2a)溶于 $200 \mathrm{~mL}$ 的冰醋酸, 加热升温至回流, 缓 慢滴加入 $23 \mathrm{~mL}$ 的 $85 \%$ 水合联胺溶于 $50 \mathrm{~mL}$ 醋酸的溶 液, 保温摚拌反应 $5 \mathrm{~h}$, 冷却到室温, 抽滤, 滤饼用乙醇 洗, 滤液减压浓缩干, 向残余物内加入 $200 \mathrm{~mL}$ 水搅拌 分散, 抽滤, 滤饼用无水乙醇重结晶, 得到 $14.5 \mathrm{~g}$ 的 2,7二溴-4,5-二氟菲(1a), 白色片状结晶, 产率 $85 \%$. ${ }^{1} \mathrm{H}$ NMR $\left(\mathrm{CDCl}_{3}, 300 \mathrm{MHz}\right) \delta: 7.46 \sim 7.50(\mathrm{~m}, 2 \mathrm{H}), 7.56(\mathrm{~s}$, $2 \mathrm{H}), 7.76 \sim 7.77(\mathrm{~d}, J=1.8 \mathrm{~Hz}, 2 \mathrm{H}) ;{ }^{13} \mathrm{C} \mathrm{NMR}\left(\mathrm{CDCl}_{3}\right.$, $300 \mathrm{MHz}) \delta: 114.30,117.93,118.13,118.32,121.00$, 127.36, 128.21, 135.91; EI-MS (70 V) $m / z(\%): 372\left(\mathrm{M}^{+}\right.$, 70), 212 (100), 106 (72).

2-溴-4,5-二氟菲(12a), 白色片状结晶，产率 $85 \%$. 3.2 .102 -溴-4,5-双 (三氟甲基)-9,10-二氢菲(17b)和 2,7-二溴-4,5-双(三氟甲基)-9,10-二氢菲(18b) 的合成

$3.16 \mathrm{~g}$ (10 mmol)的 4,5-双(三氟甲基)-9,10-二氢菲 (16b)溶解在 $150 \mathrm{~mL}$ 的氯仿中, 加入 $16.2 \mathrm{mg}(0.1 \mathrm{mmol})$ 的无水氯化铁, 加热升温至回流, 缓慢滴加入 $1.95 \mathrm{~g} \mathrm{(12}$ $\mathrm{mmol}$ )的溴素溶于 $20 \mathrm{~mL}$ 氯仿的溶液, 继续回流反应 24 $h$, 冷却到室温, 用饱和的亚硫酸氢钠水溶液洗 3 次, 收 集到的有机相用无水硫酸钠干燥, 过滤, 滤液减压浓缩 干, 残余物用短的硅胶柱脱色, 得 $3.75 \mathrm{~g}$ 的 2-溴- 4,5-双 (三氟甲基)-9,10-二氢菲(17b)和 4,5-双(三氟甲基)-9,10二氢菲 $(\mathbf{1 6 b})$ 混合物, 无法分离纯化, 产物 $\mathrm{GC}$ 含量为 $79 \%$ ，黄色油状物，产率 75\%.

2,7-二溴-4,5-双(三氟甲基)-9,10-二氢菲(18b)，白色 结晶, 产率 $88 \%$.

\section{2 .11 目标化合物 19 的合成}

$10 \mathrm{mmol}$ 的中间体 $\mathbf{1}, \mathbf{1 2}, \mathbf{1 7}, \mathbf{1 8}$ 和 $11 \mathrm{mmol}$ 的芳基 硼酸 $\mathrm{Ar}^{1} \mathrm{~B}(\mathrm{OH})_{2}, 4.2 \mathrm{~g}$ 的无水碳酸钠混合, 加入 $60 \mathrm{~mL}$ 的甲苯和 $30 \mathrm{~mL}$ 的水以及 $10 \mathrm{~mL}$ 的乙醇, 再加入 $115 \mathrm{mg}$ 的 $\operatorname{Pd}\left(\mathrm{PPh}_{3}\right)_{4}$ 催化剂, 在氮气保护下, 升温回流反应过 夜, 冷却到室温, 加入 $60 \mathrm{~mL}$ 的水, 分出有机相, 用饱 和食盐水洗, 有机相用无水 $\mathrm{MgSO}_{4}$ 干燥, 过滤, 滤液减 压浓缩干，残余物用硅胶柱分离纯化，再用石油醚重结 晶，得中间体或产物 19.

4,5-二氟-2-(3,4,5-三氟苯基)菲(19_1): 白色固体， 收率 86.5\%. ${ }^{1} \mathrm{H}$ NMR $\left(\mathrm{CDCl}_{3}, 300 \mathrm{MHz}\right) \delta: 7.32 \sim 7.42$ $(\mathrm{m}, 3 \mathrm{H}), 7.50(\mathrm{~s}, 2 \mathrm{H}), 7.60 \sim 7.82(\mathrm{~m}, 4 \mathrm{H}) ;{ }^{13} \mathrm{C} \mathrm{NMR}$ $\left(\mathrm{CDCl}_{3}, 300 \mathrm{MHz}\right) \delta: 111.17,111.38,112.25,112.59$, $114.35,114.67,119.64,121.62,124.04,126.96,127.36$, 128.27, 128.36, 128.76, 134.76, 135.10; EI-MS (70 V) $\mathrm{m} / \mathrm{z}$ (\%): $344\left(\mathrm{M}^{+}, 100\right), 324$ (16), 172 (18). Anal. calcd for $\mathrm{C}_{20} \mathrm{H}_{9} \mathrm{~F}_{5}$ : C 69.77, H 2.63, F 27.59; found C 69.83, H 2.69, F 27.56 .

2-(2,3-二氟-4-正丙基苯基)-4,5-二氟菲(19_2): 白色 固体，收率 $80.6 \% .{ }^{1} \mathrm{H} \mathrm{NMR}\left(\mathrm{CDCl}_{3}, 300 \mathrm{MHz}\right) \delta: 1.01(\mathrm{t}$, $J=14.7 \mathrm{~Hz}, 3 \mathrm{H}), 1.67 \sim 1.75(\mathrm{~m}, 2 \mathrm{H}), 2.71(\mathrm{t}, J=15 \mathrm{~Hz}$, 2H), $7.06(\mathrm{t}, J=15 \mathrm{~Hz}, 1 \mathrm{H}), 7.25$ (t, $J=15 \mathrm{~Hz}, 1 \mathrm{H}), 7.37$ $(\mathrm{d}, J=8.4 \mathrm{~Hz}, 2 \mathrm{H}), 7.52 \sim 7.63(\mathrm{~m}, 2 \mathrm{H}), 7.76 \sim 7.88(\mathrm{~m}$, $3 \mathrm{H}) ;{ }^{13} \mathrm{C} \mathrm{NMR}\left(\mathrm{CDCl}_{3}, 300 \mathrm{MHz}\right) \delta: 13.86,23.29,30.90$, $114.13,115.56,115.97,123.85,124.28,125.19$, 125.25, $126.54,126.67,127.55,127.89,131.59,131.76,134.54$, 134.60, 134.69. Anal. calcd for $\mathrm{C}_{23} \mathrm{H}_{16} \mathrm{~F}_{4}$ : C 74.99, H 4.38, F 20.63; found C 74.65, H 4.39, F 20.65.

4,5-双(三氟甲基)-2-(3,4,5-三氟苯基)-9,10-二氢菲 (19_3): 白色固体，收率 78.4\%. ${ }^{1} \mathrm{H} \mathrm{NMR}\left(\mathrm{CDCl}_{3}, 300\right.$ $\mathrm{MHz}) \delta: 2.59 \sim 2.63(\mathrm{~m}, 2 \mathrm{H}), 2.83 \sim 2.90(\mathrm{~m}, 2 \mathrm{H}), 7.29 \sim$ $7.31(\mathrm{~m}, 2 \mathrm{H}), 7.43 \sim 7.50(\mathrm{~m}, 2 \mathrm{H}), 7.59(\mathrm{~d}, J=6.9 \mathrm{~Hz}$, 2H), 7.67 (s, 1H); ${ }^{13} \mathrm{C} \mathrm{NMR}\left(\mathrm{CDCl}_{3}, 300 \mathrm{MHz}\right) \delta: 29.79$, $111.12,111.18,111.27,111.38,111.47,112.70,113.04$, $117.79,118.16,120.83,120.96,121.80,126.49,126.53$, $127.26,128.64,135.08,135.74,135.79$. Anal. calcd for 
$\mathrm{C}_{22} \mathrm{H}_{11} \mathrm{~F}_{9}$ : C 59.20, H 2.48, F 38.31; found C 59.42, H 2.55, F 38.21 .

2-(2,3-二氟-4-正戊基苯基)-4,5-双 (三氟甲基)-9,10二氢菲(19_4): 白色固体, 收率 69.8\%. ${ }^{1} \mathrm{H}$ NMR $\left(\mathrm{CDCl}_{3}\right.$, $300 \mathrm{MHz}) \delta: 0.95(\mathrm{t}, J=6.6 \mathrm{~Hz}, 3 \mathrm{H}), 1.38 \sim 1.40(\mathrm{~m}, 4 \mathrm{H})$, $1.64 \sim 1.70(\mathrm{~m}, 2 \mathrm{H}), 2.71 \sim 2.73(\mathrm{~m}, 6 \mathrm{H}), 6.87 \sim 6.96(\mathrm{~m}$, $1 \mathrm{H}), 7.07 \sim 7.33(\mathrm{~m}, 4 \mathrm{H}), 7.64 \sim 7.78(\mathrm{~m}, 3 \mathrm{H}) ;{ }^{13} \mathrm{C} \mathrm{NMR}$ $\left(\mathrm{CDCl}_{3}, 300 \mathrm{MHz}\right) \delta: 14.13,22.63,30.15,30.89,31.52$, $35.55,114.65,123.92,124.03,124.15,132.13,133.26$, 135.17, 137.09, 139.15, 140.16, 145.36, 148.38. Anal. calcd for $\mathrm{C}_{27} \mathrm{H}_{22} \mathrm{~F}_{8}$ : C 65.06, H 4.45, F 30.49; found $\mathrm{C}$ 65.11, H 4.54, F 30.37 .

\section{2 .12 目标化合物 $\mathbf{2 0}$ 的合成}

$10 \mathrm{mmol}$ 的中间体 $19\left(\mathrm{R}^{2}=\mathrm{Br}\right)$ 和 $12 \mathrm{mmol}$ 的芳基硼 酸 $\operatorname{Ar}^{1} \mathrm{~B}(\mathrm{OH})_{2}, 4.2 \mathrm{~g}$ 的无水碳酸钠混合, 加入 $60 \mathrm{~mL}$ 的 甲苯和 $30 \mathrm{~mL}$ 的水以及 $10 \mathrm{~mL}$ 的乙醇, 再加入 $115 \mathrm{mg}$ 的 $\mathrm{Pd}\left(\mathrm{PPh}_{3}\right)_{4}$ 催化剂, 在氮气保护下, 升温回流反应过 夜, 冷却到室温, 加入 $60 \mathrm{~mL}$ 的水, 分出有机相, 用饱 和食盐水洗, 有机相用无水 $\mathrm{MgSO}_{4}$ 干燥, 过滤, 滤液减 压浓缩干, 残余物用硅胶柱分离纯化, 再用石油醚重结 晶, 得目标化合物 $\mathbf{2 0}$.

4,5-二氟-2-(4-(4-正丙基环已基)苯基)-7-(3,4,5-三氟 苯基)菲(20_1): 白色固体, 收率 87.9\%. ${ }^{1} \mathrm{H} \mathrm{NMR}\left(\mathrm{CDCl}_{3}\right.$, $300 \mathrm{MHz}) \delta$ : $1.03(\mathrm{t}, J=14.8 \mathrm{~Hz}, 3 \mathrm{H}), 1.05 \sim 1.11(\mathrm{~m}$, $2 \mathrm{H}), 1.23(\mathrm{t}, J=0.78 \mathrm{~Hz}, 2 \mathrm{H}), 1.34 \sim 1.38(\mathrm{~m}, 3 \mathrm{H}), 1.48 \sim$ $1.50(\mathrm{~m}, 1 \mathrm{H}), 1.92(\mathrm{dd}, J=6.6,6.0 \mathrm{~Hz}, 4 \mathrm{H}), 2.54(\mathrm{t}, J=$ $12.3 \mathrm{~Hz}, 1 \mathrm{H}), 7.34$ (t, $J=0.6 \mathrm{~Hz}, 4 \mathrm{H}), 7.52(\mathrm{~d}, J=6.3 \mathrm{~Hz}$, 1H), $7.63 \sim 7.68(\mathrm{~m}, 3 \mathrm{H}), 7.71 \sim 7.73(\mathrm{~m}, 1 \mathrm{H}), 7.77(\mathrm{~d}, J=$ $1.8 \mathrm{~Hz}, 2 \mathrm{H}), 7.86(\mathrm{~s}, 1 \mathrm{H}) ;{ }^{13} \mathrm{C} \mathrm{NMR}\left(\mathrm{CDCl}_{3}, 300 \mathrm{MHz}\right) \delta$ : $14.51,20.14,33.64,34.40,37.13,39.81,44.45,111.12$, $111.18,111.21,111.30,111.41,112.29,112.63,113.05$, $113.37,121.63,127.14,127.67,128.69,135.30,135.37$, $136.49,141.18,141.29,148.34,161.30$. Anal. calcd for $\mathrm{C}_{35} \mathrm{H}_{29} \mathrm{~F}_{5}$ : C 77.19, H 5.37, F 17.44; found C 77.26, H 5.42, F 17.32 .

2-[3,5-二氟-4-(三氟甲氧基)苯基]-7-(2,3-二氟-4-正 丙基苯基)-4,5-二氟菲(20_2), 白色固体, 收率 67.6\%. ${ }^{1} \mathrm{H}$ NMR $\left(\mathrm{CDCl}_{3}, 300 \mathrm{MHz}\right) \delta: 1.01(\mathrm{t}, J=14.7 \mathrm{~Hz}, 3 \mathrm{H})$, $1.67 \sim 1.75(\mathrm{~m}, 2 \mathrm{H}), 2.73(\mathrm{t}, J=15.0 \mathrm{~Hz}, 2 \mathrm{H}), 7.06(\mathrm{t}, J=$ $15.0 \mathrm{~Hz}, 1 \mathrm{H}), 7.24$ (t, $J=14.7 \mathrm{~Hz}, 1 \mathrm{H}), 7.37$ (d, $J=8.4 \mathrm{~Hz}$, $2 \mathrm{H}$ ), $7.58(\mathrm{q}, J=32.4 \mathrm{~Hz}, 2 \mathrm{H}), 7.76 \sim 7.87(\mathrm{~m}, 4 \mathrm{H}) ;{ }^{13} \mathrm{C}$ NMR $\left(\mathrm{CDCl}_{3}, 300 \mathrm{MHz}\right) \delta: 13.73,24.16,31.09,111.12$, $111.36,117.63,123.37,127.36,129.72,131.06,135.41$, 139.13, 139.21, 145.86, 145.95, 154.27. Anal. calcd for $\mathrm{C}_{30} \mathrm{H}_{17} \mathrm{~F}_{9} \mathrm{O}: \mathrm{C}$ 63.84, H 3.04, F 30.29; found C 63.55, H
3.06, F 30.42 .

4,5-二氟-2-(2-氟-4-正戊基苯基)-7-(3,4,5-三氟苯基) 菲(20_3): 白色固体, 收率 74.8\%. ${ }^{1} \mathrm{H}$ NMR $\left(\mathrm{CDCl}_{3}, 300\right.$ MHz) $\delta: 0.92(\mathrm{t}, J=12.9 \mathrm{~Hz}, 3 \mathrm{H}), 1.36 \sim 1.39(\mathrm{~m}, 4 \mathrm{H})$, $1.65 \sim 1.70(\mathrm{~m}, 2 \mathrm{H}), 2.67(\mathrm{t}, J=15.3 \mathrm{~Hz}, 2 \mathrm{H}), 7.06(\mathrm{t}, J=$ $24.0 \mathrm{~Hz}, 2 \mathrm{H}), 7.33$ (t, $J=14.7 \mathrm{~Hz}, 2 \mathrm{H}), 7.45 \sim 7.51(\mathrm{~m}$, 2H), $7.63(\mathrm{~d}, J=12.9 \mathrm{~Hz}, 1 \mathrm{H}), 7.69 \sim 7.74(\mathrm{~m}, 3 \mathrm{H}), 7.82$ $(\mathrm{s}, 1 \mathrm{H}) ;{ }^{13} \mathrm{C}$ NMR $\left(\mathrm{CDCl}_{3}, 300 \mathrm{MHz}\right) \delta: 14.13,22.63$, $30.89,31.52,35.55,111.16,111.25,111.45,112.33$, $112.69,114.88,116.14,116.43,121.67,124.01,124.87$, $127.65,128.62,130.29,130.34,135.34,135.40,145.86$, 145.96, 158.27. Anal. calcd for $\mathrm{C}_{31} \mathrm{H}_{22} \mathrm{~F}_{6}$ : C 73.22, H 4.36, F 22.42; found C 73.54, H 4.38, F 22.32.

2,7-双(2,3-二氟-4-正丙基苯基)-4,5-二氟菲(20_4): 白色固体, 收率 $87.5 \% .{ }^{1} \mathrm{H}$ NMR $\left(\mathrm{CDCl}_{3}, 300 \mathrm{MHz}\right) \delta$ : $1.00(\mathrm{t}, J=17.6 \mathrm{~Hz}, 6 \mathrm{H}), 1.66 \sim 1.74(\mathrm{~m}, 4 \mathrm{H}), 2.71(\mathrm{t}, J=$ $14.6 \mathrm{~Hz}, 4 \mathrm{H}), 7.04(\mathrm{t}, J=15.6 \mathrm{~Hz}, 2 \mathrm{H}), 7.40 \sim 7.53(\mathrm{~m}$, $2 \mathrm{H}), 7.76(\mathrm{~s}, 2 \mathrm{H}), 7.82 \sim 7.87(\mathrm{~m}, 2 \mathrm{H}), 7.89(\mathrm{~s}, 2 \mathrm{H}) ;{ }^{13} \mathrm{C}$ NMR $\left(\mathrm{CDCl}_{3}, 300 \mathrm{MHz}\right) \delta: 13.86,23.29,30.90,114.13$, $114.25,114.37,114.48,114.71,115.52,115.56,115.97$, $123.85,123.89,123.98,124.28,125.19,125.25,126.54$, $126.27,127.55,127.89,131.59,131.76,134.54,134.60$, 134.96. Anal. calcd for $\mathrm{C}_{32} \mathrm{H}_{24} \mathrm{~F}_{6}$ : C 73.56, H 4.63, F 21.82; found C 73.82, H 4.64, F 21.76 .

2-(2-氟-4-正戊基苯基)-4,5-双 (三氟甲基)-7-(3,4,5三氟苯基)-9,10-二氢菲(20_5): 白色固体, 收率 58\%. ${ }^{1} \mathrm{H}$ NMR $\left(\mathrm{CDCl}_{3}, 300 \mathrm{MHz}\right) \delta: 0.93 \sim 0.95(\mathrm{~m}, 3 \mathrm{H}), 1.38 \sim$ $1.40(\mathrm{~m}, 4 \mathrm{H}), 1.79 \sim 1.83(\mathrm{~m}, 2 \mathrm{H}), 2.71 \sim 2.73(\mathrm{~m}, 6 \mathrm{H})$, $6.79 \sim 6.84(\mathrm{~m}, 1 \mathrm{H}), 7.07 \sim 7.33(\mathrm{~m}, 4 \mathrm{H}), 7.64 \sim 7.78(\mathrm{~m}$, $4 \mathrm{H}) ;{ }^{13} \mathrm{C} \mathrm{NMR}\left(\mathrm{CDCl}_{3}, 300 \mathrm{MHz}\right) \delta: 14.15,20.74,31.06$, 31.12 , 34.35, 111.14, 111.16, 111.21, 111.42, 112.06, $113.36,124.25,124.32,127.61,128.12,134.17,134.29$, 135.24, 135.29, 135.37, 136.34, 141.21, 141.33, 148.16 . Anal. calcd for $\mathrm{C}_{33} \mathrm{H}_{24} \mathrm{~F}_{10}$ : C 64.92, H 3.96, F 31.12; found C 65.18, H 3.72, F 31.08.

\section{2 .13 目标化合物 22 的合成}

$10 \mathrm{mmol}$ 的中间体 $\mathbf{1 9}\left(\mathrm{R}^{2}=\mathrm{Br}\right)$ 溶解在 $50 \mathrm{~mL}$ 的干燥 四氢呋喃中, 在氮气保护下, 用液氮一乙醇浴降温至 $-78{ }^{\circ} \mathrm{C}$, 缓慢滴加入 $4.8 \mathrm{~mL}$ 的 $2.5 \mathrm{~mol} / \mathrm{L}$ 正丁基锂-己 烷溶液, 控温在 $-70{ }^{\circ} \mathrm{C}$ 以下搅拌反应 $10 \mathrm{~min}$, 滴加入 $1.68 \mathrm{~g}$ 的4-丙基环已酩溶于 $15 \mathrm{~mL}$ 无水四氢呋喃的溶液, 缓慢升到室温搅拌反应 $1 \mathrm{~h}$, 滴加入 $20 \mathrm{~mL}$ 的水淬灭反 应, 分出有机相, 水相用乙酸乙酯萃取 3 次, 收集有机 相并用饱和食盐水洗, 无水 $\mathrm{MgSO}_{4}$ 干燥, 过滤, 滤液减 压浓缩干, 残余物用硅胶柱分离纯化, 得中间体 21. 
$5 \mathrm{mmol}$ 的中间体 21 溶解在 $50 \mathrm{~mL}$ 的干燥二氯甲烷 中, 在氮气保护下, 用液氮-乙醇浴降温至 $-30{ }^{\circ} \mathrm{C}$, 缓 慢滴加入 $1.75 \mathrm{~mL}(11 \mathrm{mmol})$ 的三乙基硅烷, 控温在 -30 ${ }^{\circ} \mathrm{C}$ 以下搅拌反应 $10 \mathrm{~min}$, 滴加入 $11 \mathrm{mmol}$ 的三氟化硼乙醚溶液, 在一 $30{ }^{\circ} \mathrm{C}$ 以下搅拌反应 $2 \mathrm{~h}$, 缓慢升到室温, 滴加入 $50 \mathrm{~mL}$ 饱和碳酸氢钠水溶液淬灭反应, 分出有机 相, 水相用乙酸乙酯萃取 3 次, 收集有机相并用饱和食 盐水洗, 无水 $\mathrm{MgSO}_{4}$ 干燥, 过滤, 滤液减压浓缩干, 残 余物用硅胶柱分离纯化, 再用无水乙醇重结晶, 得目标 化合物 22.

4,5-二氟-2-(4-正丙基-反式环已基)-7-(3,4,5-三氟苯 基)菲(22_1)：白色固体，收率 64.5\%. ${ }^{1} \mathrm{H}$ NMR $\left(\mathrm{CDCl}_{3}\right.$, $300 \mathrm{MHz}) \delta: 0.86(\mathrm{t}, J=14.8 \mathrm{~Hz}, 3 \mathrm{H}), 1.25 \sim 1.36(\mathrm{~m}$, $2 \mathrm{H}), 1.40 \sim 1.42(\mathrm{~m}, 2 \mathrm{H}), 1.44 \sim 1.48(\mathrm{~m}, 3 \mathrm{H}), 1.50 \sim 1.54$ $(\mathrm{m}, 2 \mathrm{H}), 1.88 \sim 2.00(\mathrm{~m}, 4 \mathrm{H}), 2.52 \sim 2.56(\mathrm{~m}, 1 \mathrm{H}), 7.32 \sim$ $7.34(\mathrm{~m}, 2 \mathrm{H}), 7.48(\mathrm{~d}, J=12.0 \mathrm{~Hz}, 1 \mathrm{H}), 7.61(\mathrm{~s}, 2 \mathrm{H})$, $7.63 \sim 7.67(\mathrm{~m}, 2 \mathrm{H}), 7.77(\mathrm{~s}, 1 \mathrm{H}) ;{ }^{13} \mathrm{C}$ NMR $\left(\mathrm{CDCl}_{3}, 300\right.$ MHz) $\delta: 14.52,20.15,33.63,34.42,37.13,39.82$, 44.46, $112.12,112.27,114.70,115.16,122.64,125.06,128.25$, $128.62,135.31,135.38,135.47,138.14$. Anal. calcd for $\mathrm{C}_{29} \mathrm{H}_{25} \mathrm{~F}_{5}$ : C 74.35, H 5.38, F 20.28; found C 74.65, H 5.42, F 20.16 .

2-(4-正丙基-反式环已基)-4,5-双(三氟甲基)-7(3,4,5-三氟苯基)-9,10-二氢菲(22_2): 白色固体，收率 52.7\%. ${ }^{1} \mathrm{H} \mathrm{NMR}\left(\mathrm{CDCl}_{3}, 300 \mathrm{MHz}\right) \delta$ : $0.95(\mathrm{t}, J=15 \mathrm{~Hz}$, $3 \mathrm{H}), 1.05 \sim 1.11(\mathrm{~m}, 4 \mathrm{H}), 1.22 \sim 1.51(\mathrm{~m}, 5 \mathrm{H}), 1.89 \sim 1.96$ $(\mathrm{m}, 4 \mathrm{H}), 2.71 \sim 2.83(\mathrm{~m}, 5 \mathrm{H}), 7.21 \sim 7.25(\mathrm{~m}, 4 \mathrm{H}), 7.28 \sim$ $7.32(\mathrm{~m}, 1 \mathrm{H}), 7.67(\mathrm{~s}, 1 \mathrm{H}) ;{ }^{13} \mathrm{C} \mathrm{NMR}\left(\mathrm{CDCl}_{3}, 300 \mathrm{MHz}\right) \delta$ : $14.45,20.17,31.06,31.14,33.74,34.51,37.16,39.80$, $44.55,111.12,111.18,111.24,111.47,112.14,122.63$, $124.26,127.67,128.68,134.22,134.29,135.45,135.49$, 141.34, 148.49. Anal. calcd for $\mathrm{C}_{31} \mathrm{H}_{27} \mathrm{~F}_{9}$ : C 65.26, H 4.77,
F 29.97; found C 65.65, H 4.80, F 29.86.

\section{References}

[1] Xu, H. Ph.D. Dissertation, Jilin University, Changchun, 2009 (in Chinese). (许海, 博士论文, 吉林大学, 长春, 2009.)

[2] Dubois, F.; Gingras, M. Tetrahedron Lett. 1998, 39, 5039.

[3] Kharasch, M. S.; Nudenberg, W.; Fields, E. K. J. Am. Chem. Soc. 1944, 66, 1276.

[4] Campeau, L. C.; Parisien, M.; Jean, A.; Fagnou, K. J. Am. Chem. Soc. 2006, 128, 581.

[5] Brown, C.; Sikkel, B. J.; Carvalho, C. F.; Sargent, M. V. J. Chem Soc., Perk. Trans. 1 1982, 3007.

[6] Shi, M.; Xu, B. J. Org. Chem. 2002, 67, 294.

[7] Kuninobu, Y.; Tatsuzaki, T.; Matsuki, T.; Takai, K. J. Org. Chem. 2011, 76, 7005.

[8] Some, S.; Dutta, B.; Ray, J. K. Tetrahedron Lett. 2006, 47, 1221.

[9] Bacon, L. J. Chem. Soc. 1958, 1375.

[10] Cosmo, R.; Sternhell, S. Aust. J. Chem. 1987, 40, 35.

[11] Yosuke, N.; Takahiro, F.; Sachiko, S.; Jun, N. Chem. Lett. 1999, 10, 1039.

[12] Tian, H.; Shi, J.; Dong, S.; Yan, D.; Wang, L.-X.; Geng, Y.; Wang, F. S. Chem. Commun. 2006, 33, 3498.

[13] Cosmo, R.; Sternhell, S. Australian J. Chem. 1987, 40, 2137.

[14] Witting, G.; Zimmermann, H. Chem. Ber. 1953, 86, 629.

[15] Newman, M. S.; Lilje, K. C. J. Org. Chem. 1979, 44, 4944.

[16] Ramurthy, S.; Lin, X.; Subramanian, S.; Rico, A. C.; Wang, X. M.; Jain, R.; Murray, J. M.; Bashman, S. E.; Warne, R. L.; Shu, W.; Zhou, Y.; Dove, J.; Aikawa, M.; Amiri, P.; Wang, W.; Jensen, J. M.; Wagman, A. S.; Pfister, K. B.; Ng, S. C. WO 2007117607, 2007 [Chem. Abstr. 2007, 147, 469365].

[17] Ligtenbarg, A. G. J.; Beuken, E. K.; Meetsma, A.; Veldman, N.; Smeets, W. J. J. J. Chem. Soc. 1998, 2, 263.

[18] Dameron, A. A.; Ciszek, J. W.; Tour, J. M.; Weisc, P. S. J. Phys. Chem. B 2004, 108, 16761 .

[19] Ciszek, J. W.; Tour, J. M. Tetrahedron Lett. 2004, 45, 2801.

[20] Kawamura, M.; Funahashi, M. US 20070029927, 2007 [Chem. Abstr. 2007, 146, 216108].

[21] Helms, A.; Heiler, D.; Mclendon, G. J. Am. Chem. Soc. 1992, 114, 6227.

[22] Vonlanthen, D.; Rotzler, J.; Neuburger, M.; Mayor, M. Eur. J. Org. Chem. 2010, 75, 120.

(Qin, X.) 